\title{
Cambio de modalidad dialítica motivada por la implantación de un marcapasos gástrico
}

\author{
Beatriz Peláez Requejo, Mónica Fernández Pérez, Miguel Núñez Moral, Isabel González Díaz, Samuel Robledo \\ Antón, Reyes Fernández Díaz
}

Unidad de Diálisis Peritoneal del Hospital Central de Asturias. Oviedo. España

\section{Introducción}

La gastroparesia es una alteración crónica de la motilidad gástrica en la que se produce un retraso en el vaciamiento en ausencia de una obstrucción mecánica.

La desorganización de la peristalsis antral puede ser consecuencia de cualquier desorden que induzca una disfunción neuromuscular del tracto gastrointestinal, ya que el vaciamiento gástrico refleja la coordinación de diferentes regiones del estómago y el duodeno, así como la modulación extrínseca del sistema nervioso central ${ }^{1}$.

Las etiologías más frecuentes son la idiopática, la diabética y la postquirúrgica aunque también se contemplan otras menos habituales entre ellas las que son consecuencia de trastornos de la conducta alimentaria.

Los síntomas habituales incluyen plenitud postprandial, saciedad precoz, náuseas, vómitos, malestar abdominal y sensación de distensión. El diagnóstico de certeza tras excluir otras causas como la obstrucción intestinal, se basa en pruebas que demuestren el vaciamiento gástrico enlentecido, siendo el patrón oro el estudio con escintigrafía ${ }^{1,2}$.

Los objetivos del tratamiento son asegurar el adecuado aporte nutricional que se ve amenazado en este contexto, tratar la enfermedad subyacente si es re-

\section{Correspondencia:}

Beatriz Peláez Requejo

HUCA. Unidad de Diálisis Peritoneal

Avda. de Roma s/n, Finca La Cadellada

33011 Oviedo. Asturias. España

E-mail: beatrizpelaezr@yahoo.es conocible y aliviar la sintomatología. Como abordaje inicial son básicos los cambios en la dieta y la terapia con fármacos antieméticos y procinéticos; cuando estas medidas fracasan, los vómitos son incoercibles y derivan en alteraciones electrolíticas, es necesario ingreso hospitalario para la realización de maniobras terapéuticas más invasivas. En casos extremos se inicia nutrición enteral o parenteral, para el tratamiento farmacológico se usa la vía intravenosa y en casos de gastroparesia refractaria a tratamiento médico, se valoran la inyección intrapilórica de toxina botulínica (malos resultados) y la estimulación gástrica3,4.

Esta última consiste en la implantación de un marcapasos en el tejido subcutáneo de la pared abdominal que se conecta con la capa muscular del antro a través de unos electrodos, éstos envían impulsos eléctricos de baja intensidad y elevada frecuencia que estimulan la actividad vagal para acelerar el vaciamiento $0^{5,6}$. La colocación del estimulador se realiza normalmente con técnica laparoscópica y el generador queda en el tejido subcutáneo, no más profundo de $4 \mathrm{~cm}$ para permitir la telemetría y lo más alejado posible de otros dispositivos implantables que pueda llevar el paciente; la localización más frecuente es el cuadrante medio derecho que proporciona una ubicación cómoda para la mayoría de los pacientes ${ }^{7}$.

El estimulador Enterra Therapy System (Medtronic, Minneapolis, MN,USA) fue el único aprobado en el año 2000, en USA, como dispositivo de exención humanitaria ${ }^{8}$ y en nuestro país sólo hay 5 pacientes portadores.

Los resultados obtenidos a corto-medio plazo que presentan los estudios ${ }^{9-12}$, parecen esperanzadores, mejorando la calidad de vida de los pacientes y evitando tener que llegar como último recurso a la cirugía que se reserva a casos en los que todas las medidas anteriores han fracasado. 


\section{Caso clínico}

Presentamos el caso de una mujer de 52 años, conocida en nuestra unidad desde 2008 y que ha dado consentimiento informado para la realización de este trabajo.

Como antecedente de interés sólo destaca que fue intervenida de teratoma en 1988 con doble ooferectomía que es donde comienza una conducta purgativa por miedo a convertirse en obesa tras la menopausia quirúrgica. No hipertensión arterial ni diabetes mellitus.

En septiembre de 2006 es remitida al servicio de Nefrología desde la consulta de Atención Primaria por presencia de cifras elevadas de urea y creatinina en una analítica de rutina, llegándose al diagnóstico de fracaso renal agudo secundario a deshidratación por vómitos, aunque dadas las cifras elevadas de PTH no se descarta una insuficiencia renal crónica de base. Además durante el ingreso es vista por el servicio de Psiquiatría que ratifica el diagnóstico de bulimia nerviosa. En este momento su peso al ingreso es de $39,5 \mathrm{~kg}$ con una talla de $167 \mathrm{~cm}$ (Índice Masa Corporal:14,16) y al alta es de $44 \mathrm{Kg}$.

Se realiza biopsia renal en febrero de 2007 y se confirma insuficiencia renal crónica secundaria a nefropatía intersticial secundaria a hipopotasemia por vómitos de repetición.

Desde este momento es controlada de forma ambulatoria y en abril de 2008 se coloca catéter peritoneal para iniciar el tratamiento renal sustitutivo de su elección. Inicia terapia domiciliaria en diálisis peritoneal continua ambulatoria en mayo (realiza tres intercambios diarios con un volumen de $2000 \mathrm{ml}$, dos diurnos de glucosa $1,5 \%$ y el nocturno de glucosa $2,3 \%$ ) y así se mantiene hasta marzo de 2009 que comienza con diálisis peritoneal automatizada (terapia de 8 horas, 5 ciclos de 75 minutos de permanencia y un volumen de $2000 \mathrm{ml}$ cada intercambio, utilizando soluciones de glucosa 1,5\%). Durante este tiempo, en septiembre de 2008 se realiza paratiroidectomia por hiperparatiroidismo grave.

En agosto de 2009 ingresa por convulsión tónico-clónica en el contexto de alcalosis metabólica y una vez estabilizada se traslada al servicio de Psiquiatría donde permanece dos meses para trabajar el control de las conductas purgativas sin conseguir aumento de peso (peso al alta de $38 \mathrm{Kg}$ ). Durante este ingreso se diagnostica también de enfermedad celiaca con atrofia vellositaria grave y altos títulos de anti-transglutaminasa, una vez se realizó dieta sin glúten se normalizaron los anticuerpos, si bien persistieron los vómitos.

En enero de 2010 se realiza reparación quirúrgica de hernia inguinal izquierda que precisa descanso peritoneal de un mes durante el cual realiza hemodiálisis a través de catéter temporal. En mayo de este año se realiza un tránsito gastrointestinal que resulta normal.

En noviembre de 2010 se recambia catéter peritoneal después de 4 episodios de peritonitis y sospecha de biofilm bacteriano. Se mantiene estable en la técnica y en julio de 2012 sufre una peritonitis por Staphylococcus epidermidis con varias recidivas a pesar de realizar sellado con urokinasa y reentrenamiento. En abril de 2013 inicia profilaxis antibiótica con trimetoprima/sulfametoxazol $160 \mathrm{mg} / 800 \mathrm{mg}$ diario y se hace nuevamente el sellado, esta vez con taurolidina (2 veces por semana durante 4 semanas) y con buenos resultados ya que no presenta ninguna infección peritoneal posteriormente.

En diciembre de 2013 se le hace estudio capsuloendoscópico sin hallazgos patológicos.

En julio de 2014 ingresa en la Unidad de Trastornos de la Alimentación con carácter urgente ante un grave descalabro somático con sentimientos de pérdida de control sobre su situación y miedo a morirse en el contexto de descompensación de un trastorno de la conducta alimentaria subtipo restrictivo purgativo de evolución crónica. Refiere no inducirse el vómito pero tenerlo frecuentemente y reflujo gastroesofágico espontáneo. Ante un peso de $34 \mathrm{~kg}$ y mala tolerancia oral se inició soporte nutricional parenteral y se consiguió un aumento de peso hasta los $38 \mathrm{~kg}$. Las pruebas diagnósticas descartaron patología digestiva que justificara los vómitos y se inició el tratamiento con sulpiride. Además, por si la presión intraabdominal generada por la técnica de la diálisis influyera en la génesis de los vómitos, se suspende ésta temporalmente y pasa a realizar Hemodiálisis a través de catéter tunelizado; una vez visto que no se modifica la evolución y respetando la preferencia de la paciente, se reinicia diálisis peritoneal y se continúan los estudios. Finalmente en septiembre, durante el mismo ingreso, la gammagrafía de vaciamiento gástrico demuestra alteración de la motilidad gástrica con defectos del vaciamiento, 
por lo que se deriva a una Unidad de Trastornos de la motilidad digestiva de otro centro, por carecer el nuestro de este servicio; allí se diagnostica de gastroparesia intensa mediante manometría, iniciando en ese momento el tratamiento con eritromicina parenteral como procinético con buena respuesta clínica. Al alta en octubre pesa 48,3 Kg (Índice Masa Corporal:17,32), se pauta tratamiento con cinitaprida, con escasa respuesta, después con eritromicina oral sin respuesta y posteriormente con prucaloprida succinato, persistiendo los vómitos.

Ingresa en Nefrología en diciembre de 2014 (8 días) y en enero de 2015 (11 días) otra vez por vómitos, intolerancia gástrica y alcalosis metabólica severa. A los tres días de recibir alta médica reingresa por idénticos motivos y con un peso de $36 \mathrm{~kg}, 5 \mathrm{Kg}$ inferior al del último alta hospitalario.

En febrero de 2015 se coloca catéter permanente para hemodiálisis y se cambia definitivamente de modalidad dialítica siguiendo recomendaciones del centro de referencia que considera la diálisis peritoneal contraindicación absoluta para la colocación y posterior funcionamiento del estimulador gástrico que se plantea como mejor alternativa de tratamiento en este punto, así que en marzo de 2015 se retira catéter peritoneal previo a la implantación del marcapasos en el mes de abril de ese año. El peso en este momento ronda los $43 \mathrm{Kg}$.

Durante el tiempo que se mantiene en Diálisis Peritoneal los parámetros de adecuación y ultrafiltración están dentro de las recomendaciones de las guías, un KTV en torno a 1.9-2, UF de $1000 \mathrm{ml}$ aproximadamente además de la diuresis residual conservada hasta el último momento, una nPCR de 1.2 y albúminas séricas rondando los $3.5 \mathrm{~g} / \mathrm{dl}$. Buena calidad de vida autopercibida medida con los test Euroqol-5D y SF 36v2.

Actualmente permanece en Hemodiálisis con buena tolerancia a la técnica, sin diuresis residual, con un peso seco de $36 \mathrm{~kg}$ y una ganancia interdialítica de menos de un $\mathrm{kg}$ (incluso a veces acude por debajo del peso seco) y un KTV calculado en torno a 1.6. Hace 3 sesiones semanales de 240 minutos de duración y técnica HDF on-line. Los mismos test de autopercepción de la salud presentan peores calificaciones.

El estimulador tiene un funcionamiento correcto, pero persiste la clínica gástrica.

\section{Discusión}

Aunque la paciente se ha mantenido durante 7 años en diálisis peritoneal con buena calidad de vida, manteniendo diuresis residual y parámetros de adecuación y ultrafiltración dentro de los límites recomendados ${ }^{13,14}$, son el agravamiento de la gastroparesia, retraso del vaciamiento gástrico y la colocación del marcapasos gástrico los que condicionan el cambio definitivo de modalidad dialítica.

A pesar de todas las medidas adoptadas, después de un año, no ha experimentado la mejoría esperada en cuanto a la clínica gástrica ni estado nutricional consecuencia de ésta y su calidad de vida autopercibida medida con los test Euroqol-5D y SF 36v2 ha empeorado, en contraste con lo que presentan otros estudios ${ }^{15}$.

Es un caso atípico, no contemplado por los fabricantes del estimulador ${ }^{16}$ ni descrito en la literatura con anterioridad que deja muchos interrogantes abiertos. Parece lógico que fuera necesario un descanso peritoneal inicial tras la implantación del dispositivo, pero no hay experiencia previa para sentenciar el abandono definitivo de la técnica y las contraindicaciones son teóricas respecto al riesgo de infección o interferencia de la presión intraabdominal en el funcionamiento del generador. De igual manera es atípica la ubicación de la pila, situado en la región central abdominal, supraumbilical, quizás con vistas a un posible trasplante renal.

Basándonos en los principios de beneficencia ${ }^{17}$ y de autonomía del paciente ${ }^{18} \mathrm{y}$ dado que no hemos encontrado en nuestra revisión bibliográfica ningún caso similar que justifique dicha postura, planteamos entonces, la pregunta de si sería posible volver a la técnica de diálisis peritoneal portando el marcapasos si fuese necesario y la paciente lo desease.

Recibido: 17 octubre 2016

Revisado: 23 octubre 2016

Modificado: 29 octubre 2016

Aceptado: 5 noviembre 2016 


\section{Bibliografía}

1. Rodríguez A, Zuleta J. De la fisiología del vaciamiento gástrico al entendimiento de la gastroparesia. Rev Col Gastroenterol. 2010;25(2):219225.

2. Luè $A$, Sopeña F. Alteraciones motoras gástricas. Medicine. 2012;11(2):97-105.

3. Hasler WL. Gastroparesis: pathogenesis, diagnosis and management. Nat Rev Gastroenterol Hepatol. 2011;8:438-453.

4. Tang DM, Friedenberg FK. Gastroparesis: approach, diagnostic evaluation, and management. Dis Mon 2011;57:74-101.

5. Soffer E, Abell T, Lin Z, Lorincz A, McCallum R, Parkman $\mathrm{H}$, et al. Review article: Gastric electrical stimulation for gastroparesis-physiological foundations, technical aspects and clinical implications. Aliment Pharmacol Ther 2009;30(7):681694.

6. Yin J, Abell TD, McCallum RW, Chen JD. Gastric neuromodulation with Enterra system for nausea and vomiting in patients with gastroparesis. Neuromodulation: Technology at the Neural Interface 2012;15(3):224-231.

7. Medtronic.com [sede web].United States; [last updated June 2016; acceso 1 de julio 2016].Enterra II Neuroestimulador model 37800 Implant manual.[1-26]Disponible en http://www.medtronic.com.

8. Humanitarian device exemption for Enterra Device.Federal Registry 2000;65:78495-78496.

9. 0'Grady G, Egbuji JU, Du P, Cheng LK, Pullan AJ, Windsor JA. High-Frequency gastric electrical stimulation for the treatment of gastroparesis: a meta-analysis. World J Surq 2009;33(8):16931701.
10. McCallum RW, Lin Z, Forster J, Roeser K, Hou Q, Sarosiek I. Gastric electrical stimulation improves outcomes of patients with gastroparesis for up to 10 years. Clinical Gastroenterology and Hepatology $2011 ; 9: 314-319: \mathrm{el}$.

11. Chu H, Lin Z, Zhong L, McCallum RW, Hou X. Treatment of high-frecuency gastric electrical stimulation for gastroparesis. J Gastroenterol Hepatol 2012;27(6):1017-1026.

12. Camilleri $M$, Parkman $H P$, Shafi $M A$, Abell $T L$, Gerson L. Clinical Guideline: Management of gastroparesis . Am J Gastroenterol 2013;108:18-37.

13. García-Pérez H, Pérez-Bañasco V, Arrieta J, Pérez-Fontán M. (2006). La prescripción de diálisis peritoneal.Evaluación de la dosis de diálisis adecuada. Nefrologia 2006;26(4):67-85.

14. Lo WK, Bargman JM., Burkart J, Krediet RT, Pollock C, Kawanishi H.ISPD. Guías clínicas/Recomendaciones.Perit Dial Int 2006;26:520-522.

15. Ross J, Masrur M, González-Heredia R, Fernando E. Effectiveness of Gastric Neurostimulation in Patients With Gastroparesis.JSLS 2014;18(3):16.

16. Medtronic.com [sede web].United States; [last updated June 2016; acceso 1 de julio 2016].Enterra Therapy Patient Guide:Enterra II.[1-26] Disponible en http://www.medtronic.com

17. Beauchamp TL, Childress JF. Principios de la ética Biomédica.Barcelona: Masson;1999.

18. Ley $41 / 2002$, de 14 de noviembre, básica reguladora de la autonomía del paciente y de derechos y obligaciones en materia de información y documentación clínica. "B0E» núm. 274, de 15 de noviembre de 2002, páginas 40126 a 40132. 